\title{
Collaborative Strategies and Simulation of Vehicle Group Behaviors for Off-Ramp Areas
}

\author{
Tong Mo, ${ }^{1}$ Keyi Li, ${ }^{1}$ Junjie Zhang, ${ }^{1}$ Lingqiao Qin, ${ }^{2}$ Zhufei Huang, ${ }^{1}$ and Haijian Li ${ }^{1}{ }^{1}$ \\ ${ }^{1}$ Beijing Engineering Research Center of Urban Transport Operation Guarantee, Beijing University of Technology, \\ Beijing 100124, China \\ ${ }^{2}$ TOPS Laboratory, Department of Civil and Environmental Engineering, University of Wisconsin-Madison, Madison, \\ WI 53706, USA \\ Correspondence should be addressed to Haijian Li; lihaijian@bjut.edu.cn
}

Received 18 March 2020; Revised 15 November 2020; Accepted 24 November 2020; Published 23 December 2020

Academic Editor: Hesham Rakha

Copyright (C 2020 Tong Mo et al. This is an open access article distributed under the Creative Commons Attribution License, which permits unrestricted use, distribution, and reproduction in any medium, provided the original work is properly cited.

\begin{abstract}
With the increase of vehicle ownership and the rapid growth of urban traffic, the problem of congestion in the off-ramp area of the main expressway has become the main factor restricting overall section efficiency and inducing traffic accidents. This paper focuses on the problem of group collaborative lane-changing behaviors of off-ramp vehicles and through vehicles in off-ramp areas and proposes four kinds of vehicle group collaborative strategies based on different road space balance conditions. According to a three-lane expressway scene, a VISSIM-based simulation model is built and the optimization scheme is simulated and evaluated. The simulation results show that with the increase of traffic flow in off-ramp areas, a flow-balance strategy for downstream lanes where off-ramp vehicles merge with the outside lane in advance is more advantageous. When vehicles are leaving the main road, if traffic flow is heavy, the flow-balance strategy for lanes where off-ramp vehicles merge with the outside lane in advance (for example, the proportion of off-ramp vehicles in three lanes is $0: 0: 1$ ) is better; otherwise, when the traffic flow on the main road is relatively small, the flow-balance strategy for lanes where off-ramp vehicles are distributed in lanes with different ratios (e.g., $1: 3: 6$ ) is better. What is more, for future traffic management in connected vehicle environments, it can be concluded that collaborative vehicle lane-changing strategies with different traffic flow states can help to enhance traffic efficiency.
\end{abstract}

\section{Introduction}

With an increase in traffic volume in off-ramp areas, the lane-changing behavior of vehicles in the deceleration lane on the expressway often causes the mainline traffic flow disorder. Therefore, off-ramp areas are easy to become the "bottleneck" that affects the efficiency and safety of the expressway.

For economic and environmental reasons, the expansion of existing infrastructure is not always a viable option. Therefore, traffic control has been proposed and used as an effective strategy to alleviate highway congestion problems [1]. Domestic and international scholars have studied the extremely complex traffic flow in ramp areas and deeply discussed control methods and models including ramp metering, mainline metering, routing control strategy, and lane allocation. The main purpose of these strategies is to improve traffic conditions by properly adjusting the inflow of on-ramps or from one section of the highway to another [2]. To prevent potential queue spillover, Yang et al. [3] introduced the off-ramp queue estimation model. It included three primary functions: off-ramp queue estimation, arterial adaptive signal operations, and freeway off-ramp priority control, which could improve traffic control efficiency over the entire corridor. Ma et al. [4] constructed the coordinated optimal control model of mainline and ramp, which could effectively balance the uneven distribution of traffic rights between the main road and ramp in the upper reaches of the merging area and eventually reduce the average traffic delay in the road network. Aiming at reducing the total travel time and the emissions in freeway traffic networks, Pasquale et al. [5] proposed a multiclass control 
scheme, which combined routing control and ramp metering strategy. Zhao et al. [6] proposed an integrated optimization model that combined on-ramp signal control with lane assignment, which could markedly improve a weaving area capacity. Zhu et al. [7] obtained the thresholds as vehicles start queuing at off-ramp by dichotomy, control variables, and VISSIM simulation. Furthermore, they also discussed the different off-ramp control methods corresponding to the traffic flow stage. By presignal and sorting area concept, Zhao et al. [8] proposed an integrated design model to decrease traffic weaving. In this way, the road resources of off-ramp and downstream intersection integrated section could be fully utilized. Sulejic et al. [9] established a model to optimize the lane-changing distribution based on the particle swarm algorithm. Lane allocation is another effective management method to improve the operation of the weaving area. And reasonable lane allocation can make the traffic flow operate in better order. Aiming at exploring the effect of different lane allocation strategies on the weaving area system, An et al. [10] established a cellular automata model. In this model, three different lane-changing rules were considered to match the driving behaviors when the lanes were allocated.

Besides, the driver's driving behavior is another significant factor that affects the efficiency of traffic in diverging areas. Based on studying the driver's driving behaviors, researchers had the opportunity to propose the corresponding countermeasures. Spiliopoulou et al. [11] put forward three route diversion strategies to reduce recurrent freeway congestion under driver-optimal conditions. From a microscopic perspective, Zeng et al. [12] found that the lanechanging behavior has the dual rule of inducing a phase transition and maintaining the current driving state of the vehicles and also discussed the lane-changing possibility theory. Lyu et al. [13] analyzed the lane-changing characteristics and speed profiles of drivers with different genders, occupations, and experiences. This analysis provided a basis for improving the behaviors of different types of drivers. Using actual survey data from two off-ramp under dissimilar operational strategies, Gong et al. [14] analyzed the vehicle type distribution in different lanes. By a driving simulator experiment, Shi and Liu [15] found that with differentiated per-lane speed limit scenarios, the level of driving safety has been improved. Yun et al. [16] compared the data of lanechanging behaviors with and without in-vehicle navigation information from both simulator and field investigation and discussed its potential benefits in improving lane-change safety on off-ramp area. The effect varies with the traffic density and the time at which navigation information is provided for the first time. Hidas [17] introduced SITRAS, a microscopic traffic network simulation model, and presented the details of the lane-changing models including both normal (unforced) and forced or cooperative lanechanging. As our previous work, based on VISSIM, Li et al. [18] constructed several vehicle groups lane-changing strategies under a connected vehicle environment in the upstream section of ramp areas. These strategies provided a theoretical basis for group cooperative control of vehicles in ramp areas. What is more, Huang et al. [19] studied the lane- changing spacing intervals under a different volume of traffic flow and off-ramp infrastructures conditions. With different parameters of the lane-changing spacing interval, traffic volume, and ratio of off-ramp vehicles, by changing the parameters such as traffic volume, off-ramp vehicle ratio and lane-changing spacing interval, they simulated the microbehavior of the off-ramp area based on VISSIM. They also pointed out that intelligent lane management can be used to improve traffic capacity by optimizing lane-changing spacing intervals.

To sum up, most of the proposed research methods are limited to the traditional optimization of traffic engineering design and improvement of traffic management level. However, the driving behavior of traffic activity subjects is not effectively optimized, resulting in no significant improvement in traffic jams in the highway off-ramp area. With the development of intelligent transportation, the concept of active traffic management and control has become more and more mature. In the area of active traffic management, new technologies offer opportunities to make full use of the existing road resources and infrastructures. Vehicles equipped with in-car communication systems are capable of exchanging messages with the infrastructure and other vehicles. Cooperative Intelligent Transport Systems support the exchange of information between vehicles and infrastructures [20-22]. The point is a collaboration between self-driving cars, mainly vehicle formation control or platoon control [23]. In the connected automatic vehicle environment, the behavior of vehicles is consistent with traffic flow theory [24, 25]. Many scholars have made a series of ideas about this, including a new lane-change protocol [26], a traffic flow model considering the influence of car-following [27], a proactive longitudinal control merging algorithm [28], and dynamic lane signals [29]. However, few studies have addressed merging and diverging induction strategies for vehicle groups of ramp areas in the connected vehicle environment. In our previous work, based on VISSIM, we have discussed merging collaborative strategies and simulation for on-ramp areas [30]. To improve the efficiency of the off-ramp in the connected vehicle environment, this paper focuses on the problem of group collaborative lane-changing behaviors of off-ramp vehicles and through vehicles in off-ramp areas and proposes four kinds of vehicle group collaborative strategies based on different road space balance conditions.

\section{Analysis of Traffic Characteristics in Off- Ramp Areas}

The main traffic operating characteristic of the off-ramp area is that a large number of main road vehicles merge onto offramp by changing lanes. Off-ramp vehicles must complete lane-changing operations within the lengths of deceleration lanes. As traffic volumes increase, frequent lane-changing behaviors are likely to interfere with the mainline traffic flow on the expressway, resulting in turbulent traffic flow and, ultimately, bottlenecks and congestion.

The lane-changing behaviors of off-ramp vehicles in deceleration lanes are divided into two situations in general (Figure 1): 
(1) When the main road traffic flow is small, an acceptable lane-changing gap is available for off-ramp vehicles to decelerate to the critical exit speed and enter deceleration lanes eventually.

(2) When the main road traffic flow is very heavy, approaches or reaches the actual capacity of expressways, the probability to find an acceptable lane-changing gap is approximately 0 . And off-ramp vehicles can only be queued in the deceleration lane, which makes the vehicles leaving the main road behind slow down. Hence, the vehicles leaving the main road can only travel to the end of deceleration lanes and forcibly merge onto off-ramps.

The location distribution of vehicles that merge onto the off-ramp is affected by many factors, including time headway, main road traffic flow, off-ramp traffic flow, main road traffic speed, off-ramp traffic speed, road conditions, road geometry, and driver's familiarity with road conditions. The primary purpose of the deceleration lane is to allow arterial traffic to enter the off-ramp safely and comfortably. When the traffic flow of the main road is small and the time headway is large, vehicles can merge at the front of deceleration lanes. When the traffic flow is very heavy, main road vehicles can only forcibly merge onto the off-ramp at the end of deceleration lanes. In the actual situation, the driver decelerates while observing the position of the exit in the upstream section of off-ramp areas. When finding acceptable lane-changing gaps, the driver will complete the diverging after entering the deceleration lane safely and calmly.

In addition, the conflict between straight and diverging traffic is most pronounced on the outside lanes of the main road. The lane-changing behaviors of the diverging vehicles and the corresponding speed adjustment have a great influence on the traffic flow in the outside lane and inside lane. As a result, before entering the bottlenecks, vehicles in the outside lane of the upstream section tend to change lanes to the inside lane in advance to avoid interference from the vehicles that merge onto the off-ramp.

\section{Methods and Strategies}

3.1. Influences of Deceleration Lane Length. When leaving the main road, vehicles will change lanes within a certain distance from the exit. In the actual situation, when the main road traffic flow is heavy, it is difficult for vehicles in different lanes to change lanes within the scope of the deceleration lane and find the appropriate acceptable clearance to leave the main road. So, they drive extremely slowly near the outermost lane, resulting in congestion and queuing near the off-ramp.

A scientifically sound design of deceleration lane lengths is necessary to allow off-ramp vehicles to exit the main lane comfortably and safely. The $n$ lanes of the main road are named outward from the median divider as the 1st lane (innermost lane), the 2 nd lane, ..., the $n$th lane (outermost lane). We take the typical road structure of off-ramp area as the research model; its sketch is shown in Figure 2.

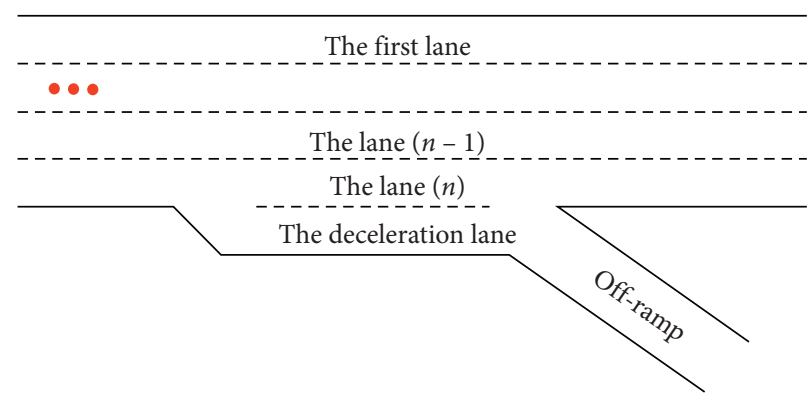

Figure 1: Typical off-ramp area sketch.

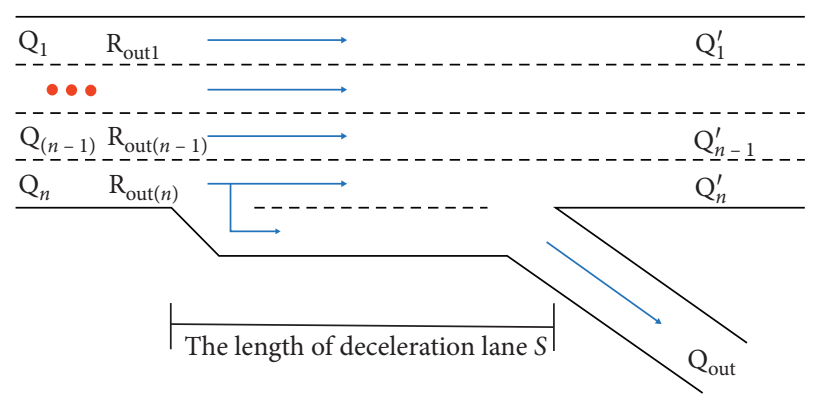

FIgURE 2: Typical off-ramp area sketch.

To find out the most appropriate deceleration lane length for off-ramp and the relationship between delays and the length of deceleration lane, we set the length of the deceleration lane to $S$. By VISSIM software, the influence of different deceleration lane lengths on vehicle delay is simulated and analyzed.

3.2. Upstream Flow-Balance Strategies. After determining the appropriate length of the deceleration lane, reasonably controlling the spatial distribution of traffic flow in the upstream and downstream of off-ramp areas is a significant issue related to the operation efficiency of off-ramp areas. By simulating the spatial distribution of different traffic flows, this paper attempts to put forward the inducing strategies that can improve road traffic efficiency under a connected vehicle environment.

For this reason, the flow-balance strategies of the upstream road section are put forward: control traffic flow of each lane of the upstream road section to be roughly equal, to ensure that the upstream road resources are fully utilized. $Q_{1}, Q_{2}, \ldots, Q_{n}$ represent the traffic flow distribution in the 1st lane (innermost lane), the 2nd lane, ..., the $n$th lane (outermost lane), respectively, of the upstream sections. $Q_{1}^{\prime}$, $Q_{2}^{\prime}, \ldots, Q_{n}^{\prime}$ represent the traffic flow distribution in the 1 st lane, the 2 nd lane, ..., the $n$th lane, respectively, of the downstream sections after vehicles change lanes in off-ramp areas. $Q_{\text {out }}$ denotes the traffic flow from the main road to the off-ramp. $R_{\text {out } 1}, R_{\text {out } 2}, \ldots, R_{\text {out } n}$ represent the proportion of off-ramp vehicles that merge leaving the 1 st lane, the 2 nd lane, ..., the $n$th lane, which satisfy the constraint $R_{\text {out } 1}+R_{\text {out } 2}+\ldots+R_{\text {out } n}=1$.

The typical off-ramp area diagram of traffic flow spatial distributions is shown in Figure 2. 
Strategy A1: Flow-balance strategies for upstream lane. Strategy A1.1: Flow-balance strategies for upstream lanes where off-ramp vehicles merge with outside lane in advance.

In upstream sections, vehicles are roughly distributed across each lane evenly, which satisfies equation (1). Off-ramp vehicles simultaneously merge onto the outside lane as a matter of priority after a lane change.

$$
Q_{1}=Q_{2}=\cdots=Q_{n}
$$

Strategy A1.2: Flow-balance strategies for upstream lanes where off-ramp vehicles are distributed in lanes with different ratios (generalized extension of strategy A1.1).

The traffic flow of each lane in upstream sections is approximately equal, which satisfies equation (1). Off-ramp vehicles, respectively, merge onto the 1st lane, the 2nd lane, $\ldots$, the $n$th lane in proportions of $R_{\text {out } 1}, R_{\text {out } 2}, \ldots, R_{\text {out } n}$, which satisfy the constraint $R_{\text {out } 1}+R_{\text {out } 2}+\cdots+R_{\text {out } n}=1$.

\subsection{Downstream Flow-Balance Strategies}

Strategy A2: Flow-balance strategies for downstream lane.

Strategy A2.1: Flow-balance strategies for downstream lanes where off-ramp vehicles merge to outside lane in advance.

The meaning of downstream flow-balance strategies is as follows: except for the vehicles leaving the main road of an expressway, the remaining straight vehicles are evenly distributed in each lane of the downstream section of off-ramp after lane transformation, that is, satisfying equation (2). At the same time, it is assumed that all vehicles leaving the main road of the expressway will change lanes ahead of time to the outermost lane of the main road, that is, satisfying equation (6). As a result, it can be deduced that the other lanes of the main road of the expressway are satisfying equations (3)-(5).

$$
\begin{aligned}
Q_{1}^{\prime} & =Q_{2}^{\prime}=\cdots=Q_{n}^{\prime}=\frac{Q_{1}+Q_{2}+\cdots+Q_{n}-Q_{\text {out }}}{n} \\
Q_{1}^{\prime} & =Q_{1}, \\
Q_{2}^{\prime} & =Q_{2}, \\
Q_{n-1}^{\prime} & =Q_{n-1}, \\
Q_{n}^{\prime} & =Q_{n}-Q_{\text {out }} .
\end{aligned}
$$

Strategy A2.2: Flow-balance strategies for downstream lanes where off-ramp vehicles are distributed in lanes with different radios (generalized extension of strategy A2.1).

$R_{\text {out } 1}, R_{\text {out } 2}, \ldots, R_{\text {out } n}$ represent the proportion of offramp vehicles that leave the 1 st lane, the 2 nd lane, ..., the $n$th lane. After lane-changing behaviors, the traffic flow of the 1st lane is composed of the original traffic flow in the 1st lane minus off-ramp traffic flow leaving the 1st lane, which satisfies equation (7). In the same way, the traffic flow of the 2nd lane and the traffic flow of the $n$th lane satisfy equations (8) and (9), respectively. According to the downstream flowbalance principle, the traffic flow of each lane in the downstream sections is approximately equal; each lane of the downstream section has roughly the same traffic volume, which satisfies equation (11). In this principle, the downstream road resources are fully utilized. By comparison, strategy A2.2 is a general case of strategy A2.1, and strategy A2.1 is considered a special case of strategy A2.2, where $R_{\text {out } 1}=0, R_{\text {out } 2}=0, \ldots, R_{\text {out }(n-1)}=0$, and $R_{\text {out } n}=1$.

$$
\begin{aligned}
& Q_{1}^{\prime}=Q_{1}-Q_{\text {out }} * R_{\text {out } 1}, \\
& Q_{2}^{\prime}=Q_{2}-Q_{\text {out }} * R_{\text {out } 2}, \\
& Q_{n}^{\prime}=Q_{n}-Q_{\text {out }} * R_{\text {out } n}, \\
& R_{\text {out } 1}+R_{\text {out } 2}+\cdots+R_{\text {out } n}=1, \\
& Q_{1}^{\prime}=Q_{2}^{\prime}=\cdots=Q_{n}^{\prime}=\frac{Q_{1}+Q_{2}+Q_{n}-Q_{\text {out }}}{n} .
\end{aligned}
$$

\section{Strategies Simulation and Evaluation Based on VISSIM}

\subsection{VISSIM Simulation Model and Parameter Settings}

4.1.1. VISSIM Simulation Model. In this paper, various collaborative strategies of vehicle groups are suggested based on the lateral spatial distributions of traffic flows and geometric lengths of deceleration lanes. Through VISSIM simulation, different strategies are compared and discussed. To facilitate understanding, a simulation model of road structure for off-ramp area with lane number $n=3$ is constructed; that is, the main expressway is three-lane and the off-ramp is one-lane. A screenshot is shown in Figure 3.

\subsubsection{Parameter Settings}

(1) Detectors setting: travel time/vehicle delay detectors spacing from the main road to the main road or from the off-ramp to the main road is $1000 \mathrm{~m}$.

(2) The downstream section of the off-ramp area is 200 meters in length.

(3) The off-ramp is $200 \mathrm{~m}$ in length.

(4) The off-ramp deceleration lane is $20-300 \mathrm{~m}$ in length.

(5) Traffic flow detection nodes setting: main road traffic flow detection nodes are located $2 \mathrm{~m}$ from their 
starting points; off-ramp traffic flow detection nodes are located $2 \mathrm{~m}$ from their starting points.

(6) Speed limit settings: $60-80 \mathrm{~km} / \mathrm{h}$ for the main road and $20-40 \mathrm{~km} / \mathrm{h}$ for off-ramp (the green triangle in Figure 4).

(7) The working distance of the lane change in advance is $400 \mathrm{~m}$.

(8) The main road has an hourly traffic flow of $1200 /$ lane.

(9) The ratio of vehicles leaving the main road is $20 \%$.

As shown in Figure 4, the green vertical lines represent the beginning of travel time detectors, the red vertical lines represent travel time detector terminals, the distance between the two is 1000 meters, the green triangles represent speed limit points, and the purple diamonds represent traffic flow detection nodes.

4.2. Influence Evaluation of Deceleration Lane Length. To study the influence characteristics of the geometric length mentioned in Section 3.1, this section analyzes the quantitative relationship between the vehicle delay and the deceleration lane length $S$ via simulation and the influence of the geometric length on the traffic characteristics of the offramp areas and driving behaviors of drivers.

The deceleration lane $S$ ranges from 20 meters to 300 meters with an interval of 20 meters. Therefore, there are 15 research situations. VISSIM is used to simulate the influence of the deceleration lane length $S$ on vehicle passage efficiency under the circumstance that the main road has an hourly traffic flow of $1200 /$ lane and the ratio of vehicles leaving the main road is $20 \%$. Two parameters of vehicle delay and travel time were used to evaluate vehicle efficiency at different deceleration lane lengths. The indexes of travel time and delay in different conditions are shown in Figures 5 and 6.

According to Figures 5 and 6, vehicle delay and travel time decrease with the increase of the length $S$ when the length $S$ of the deceleration lane is less than 80 meters; vehicle delay and travel time are approximately horizontal straight lines as the length $S$ increases when the length $S$ of the deceleration lane is greater than 80 meters; that is, the length $S$ of the deceleration lane is no longer the main factor affecting vehicle passage efficiency.

Therefore, the length of the deceleration lane should be greater than or equal to 80 meters. However, considering the actual situation, a deceleration process is needed for main road vehicles to enter the off-ramp. Generally, the speed limit of the off-ramp is $40 \mathrm{~km} / \mathrm{h}$ and the speed limit of the main road is $80 \mathrm{~km} / \mathrm{h}$. The length of the deceleration lane must at least satisfy the distance requirement of a vehicle to decelerate from 80 to $0 \mathrm{~km} / \mathrm{h}$, taking into account the queue of vehicles on the off-ramp.

The following suggestions are given to improve the capacity of the off-ramp: the expected reduction speed of a car is $3-3.5 \mathrm{~m} / \mathrm{s}^{2}$. According to $v^{2}=2 a^{*} S$, the recommended length of the deceleration lane ranges from 80 to 120 meters.

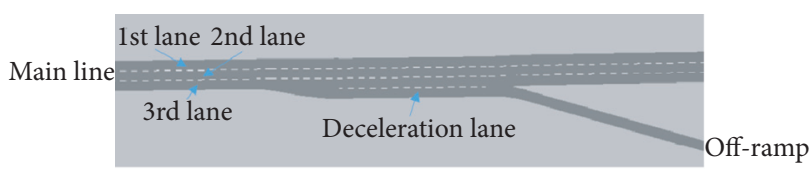

FIGURE 3: Screen capture of the VISSIM simulation model in the off-ramp area.

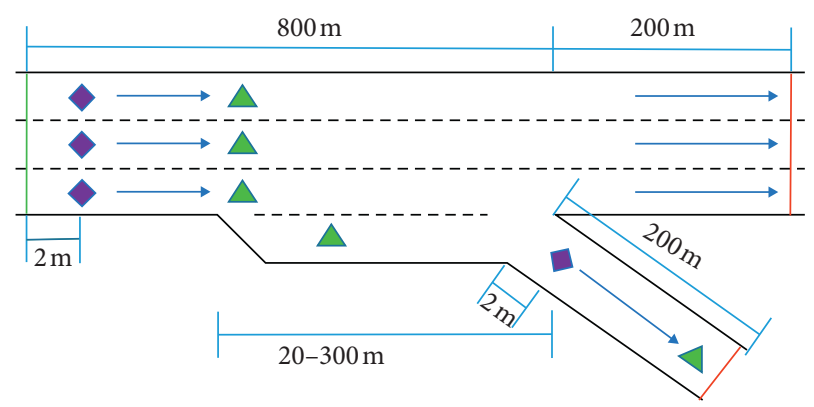

FIGURE 4: VISSIM simulation model parameter settings sketch for off-ramp area.

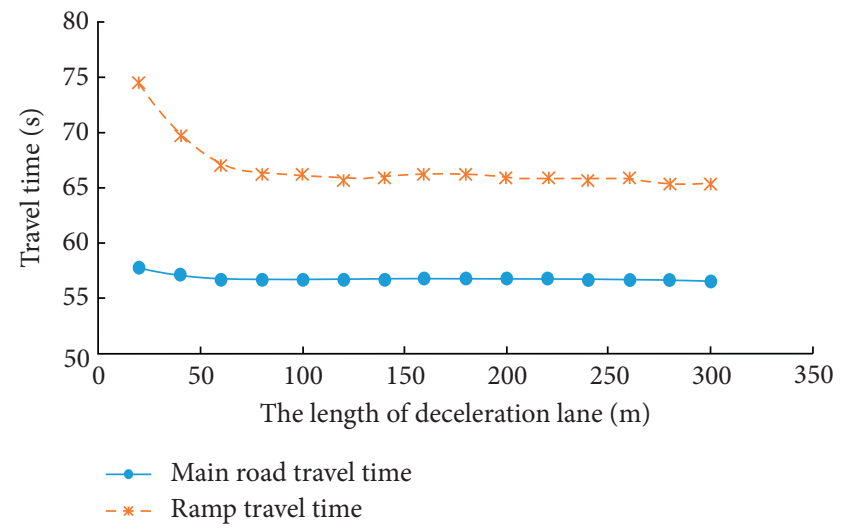

FIgURE 5: The relationship between travel time and the length of the acceleration lane.

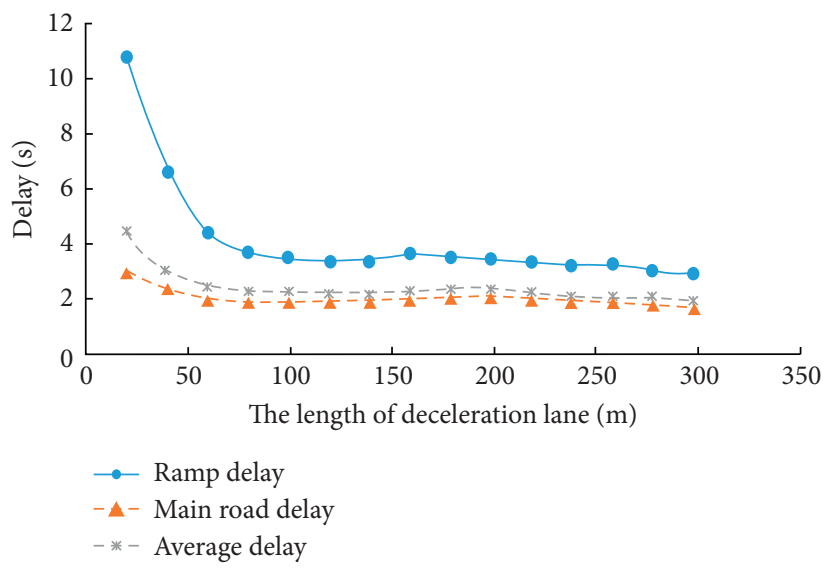

FIgURE 6: The relationship between delay and the length of the acceleration lane. 
4.3. Simulation Results of Flow-Balance Strategies for Upstream Lane. To study the flow-balance strategies for upstream lane, this section analyzes the influence of different lateral spatial distributions of traffic flow on the traffic characteristics and the driving behaviors of drivers of the offramp area through VISSIM simulation, to study the control strategy based on the lateral spatial distribution of ramp vehicle flow.

Setting $R_{\text {out } 1}=0.1, R_{\text {out } 2}=0.3$, and $R_{\text {out } 3}=0.6$, the simulation is performed in 15 cases, in which the traffic flow per lane per hour on the main road is 1000, 1200, 1400, 1600, and 1800 , respectively. The ratio of vehicles leaving the main road is $20 \%, 30 \%$, and $40 \%$, respectively. Figures 7 shows the average vehicle delay curves when using strategies A1.1 and A1.2 in different traffic flow spatial distributions.

Under most traffic conditions, the average vehicle delay value under the A1.1 strategy is lower than the average vehicle delay value under the A1.2 strategy. As the ratio of off-ramp vehicles and main road traffic gradually increases, the effect of adopting strategy A1.1 on improving vehicle passage efficiency in the off-ramp area is much better than that of strategy A1.2 in most cases. As for the A1.1 strategy, under the higher off-ramp ratio, when the traffic flow continues to increase, the delay decreases gradually. However, when the ratio of off-ramp vehicles gradually increases, and the main road traffic is still low at the same time, strategy A1.2 is slightly better than strategy A1.1.

The above analysis shows that, for the three-lane expressway scenario, when the traffic flow is low and fewer vehicles are leaving the main road, these two flow-balance strategies have similar effects. At the same time, when the traffic flow is low and the vehicles leaving the main road gradually increase, the flow-balance strategies for upstream lanes where off-ramp vehicles are distributed in lanes with different ratios are more effective (for example, the strategy of inducing vehicles in advance to be distributed in the inside lane, middle lane, and outside lane of the main road at a ratio of $1: 3: 6$ is better). When the traffic flow and the ratio of offramp vehicles increase at the same time, the flow-balance strategies for upstream lanes where off-ramp vehicles merge with outside lane in advance are more effective (for example, the strategy of inducing vehicles in advance to be distributed in the inside lane, middle lane, and outside lane of the main road at a ratio of $0: 0: 1$ is better). With more traffic flow, the advantages are even more pronounced.

4.4. Simulation Results of Flow-Balance Strategies for Downstream Lane. To study the flow-balance strategies for downstream lane, this section analyzes the influence of different lateral spatial distributions of traffic flow on the traffic characteristics and the driving behaviors of drivers of the off-ramp area through VISSIM simulation, to study the control strategy based on the lateral spatial distribution of ramp vehicle flow.

Setting $R_{\text {out } 1}=0.1, R_{\text {out } 2}=0.3$, and $R_{\text {out } 3}=0.6$, the simulation is performed in 15 cases, in which the traffic flow per hour per lane on the main road is $1000,1200,1400,1600$, and 1800 , respectively. The ratio of vehicles leaving the main road is $20 \%, 30 \%$, and $40 \%$, respectively. Figure 8 shows the average vehicle delay curves when using strategies A2.1 and A2.2 in different traffic flow spatial distributions.

Under most traffic conditions, the average vehicle delay value under the A2.1 strategy is lower than the average vehicle delay value under the A2.2 strategy. As the ratio of off-ramp vehicles and main road traffic gradually increases, the effect of adopting strategy A2.1 on improving vehicle passage efficiency in the off-ramp area is much better than that of strategy A2.2 in most cases. As for the A2.1 strategy, under the higher off-ramp ratio, when the traffic flow continues to increase, the delay decreases gradually.

The above analysis shows that, for the three-lane expressway scenario, when the traffic flow is low and fewer vehicles are leaving the main road, these two flow-balance strategies have similar effects. At the same time, when the traffic flow is low and the vehicles leaving the main road gradually increase, the flow-balance strategies for downstream lanes where off-ramp vehicles are distributed in lanes with different ratios are more effective. When the traffic flow and the ratio of off-ramp vehicles increase at the same time, the flow-balance strategies for upstream lanes where offramp vehicles merge with the outside lane in advance are more effective. With more traffic flow, the advantages are even more pronounced.

\section{Results Analysis and Discussion}

5.1. Comparative Analysis of Strategies. In this section, four strategies are analyzed and compared to determine the optimal strategy under different traffic flow environments. The comparison results are shown in Table 1. First of all, the traffic flow through the detection node is analyzed according to the traffic flow state under different conditions [30]. The comparison results are shown in Table 1.

In Table 1, numbers in roman and italics represent congestion and noncongestion, respectively. Concerning the simulation data of travel time and vehicle delays, the effectiveness of the four strategies to improve traffic efficiency under different road conditions is comparatively analyzed. More specifically, the strategies can be categorized into four main cases as follows:

(1) The bar chart in Figure 9 compares vehicle delays under four strategies when both the off-ramp rate and main road traffic flows are low (for example, hourly traffic flow per lane on the main road is 1000 , and the off-ramp rate is $20 \%$ per hour).

When both the main road traffic flow and the offramp rate are low, the effects of the four strategies are almost the same: A1.1 = A2.1 = A $1.2=\mathrm{A} 2.2$ (where " $>$ " indicates that the former is more effective than the latter in alleviating vehicle delays, while " =" indicates that the vehicle delays in the two strategies are similar).

(2) The bar chart in Figure 10 compares vehicle delays for four strategies when the off-ramp rate is high and the main road traffic flow is low (for example, hourly 


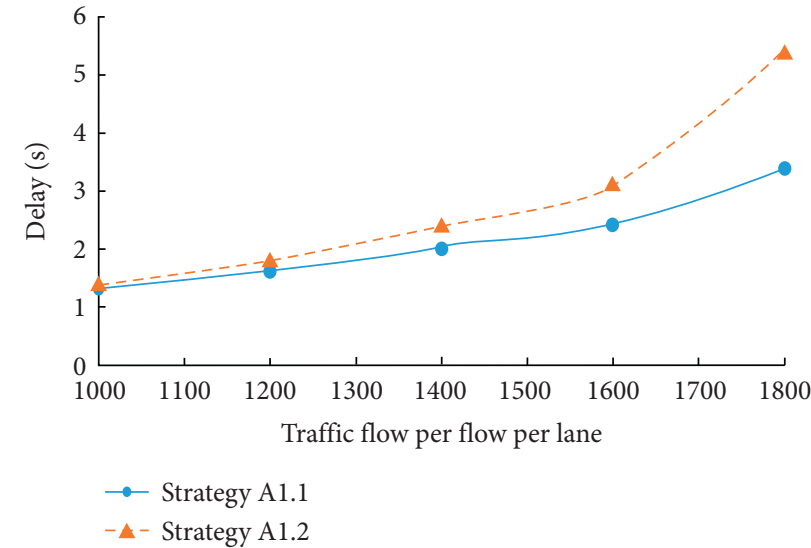

(a)

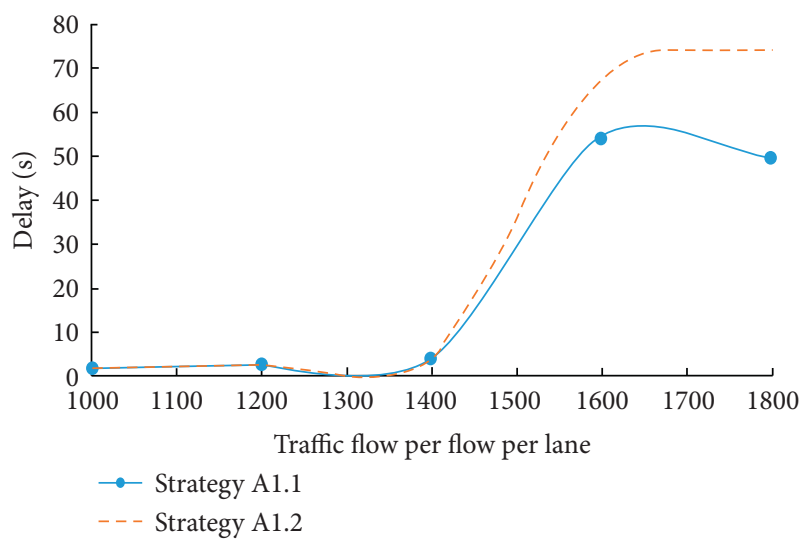

(b)

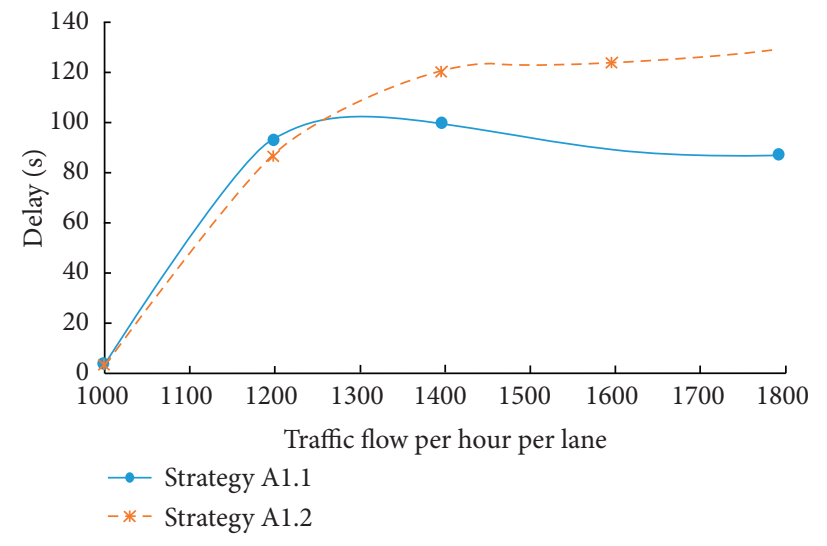

(c)

Figure 7: The average vehicle delay change curves of strategy A1.1 and strategy A1.2 at different off-ramp flow. (a) 20\%. (b) $30 \%$. (c) $40 \%$.

traffic flow per lane on the main road is 1200 , and the off-ramp rate is $40 \%$ per hour).

When the off-ramp rate is high and the main road traffic flow is low, strategies A1.2 and A2.2 have a reasonable and similar effect on alleviating vehicle delays, while strategies A1.1 and A2.1 are poor. We have $\mathrm{A} 2.2=\mathrm{A} 1.2>\mathrm{A} 2.1>\mathrm{A} 1.1$.

(3) The bar chart in Figure 11 compares vehicle delays under four strategies when both the off-ramp rate and main road traffic flows are high (for example, traffic flow per lane on the main road is 1600 , and the off-ramp rate is $30 \%$ per hour).

When both the off-ramp and the main road are in a higher traffic flow state, strategies A2.1 and A1.1 are slightly better in reducing vehicle delays. When both the main road traffic flow and the off-ramp rate are high, strategy A2.1 and strategy A1.1 are more effective in alleviating vehicle delays. We have A2.1 > A1.1 > A2.2 > A1.2.

(4) The bar chart in Figure 12 compares vehicle delays under four strategies when both the off-ramp rate and the main road traffic flows are extremely high (for example, traffic flow per lane on the main road is 1800 , and the off-ramp rate is $40 \%$ per hour).
When both the main road and the off-ramp are in heavy traffic flow conditions, strategy A2.1 has the best overall effect, followed by strategy A1.1. And there is a similar effect between strategy 1.2 and strategy 2.2. We have $\mathrm{A} 2.1>\mathrm{A} 1.1>\mathrm{A} 1.2=\mathrm{A} 2.2$.

5.2. Discussion. Different traffic conditions are divided into 6 traffic flow states in different colors. Light green indicates that both the off-ramp rate and main road traffic flows are low. Dark green means that the off-ramp traffic rate is high and the main road traffic flow is low. Yellow means that the main road traffic flow is high and the offramp rate is low. Brown means that the main road traffic flow is extremely high and the off-ramp rate is low. Pink indicates that both the off-ramp rate and main road traffic flows are high and red indicates that both the off-ramp rate and main road traffic flows are extremely high. Table 2 analyzes and compares the advantages and disadvantages of these four strategies in different traffic flow states.

According to Table 2, the average vehicle delay in the offramp area is kept to a minimum by using an optimal induction strategy for different traffic flow states.

A three-dimensional bar chart of optimal average vehicle delays is shown in Figure 13. The optimal strategy in the light 


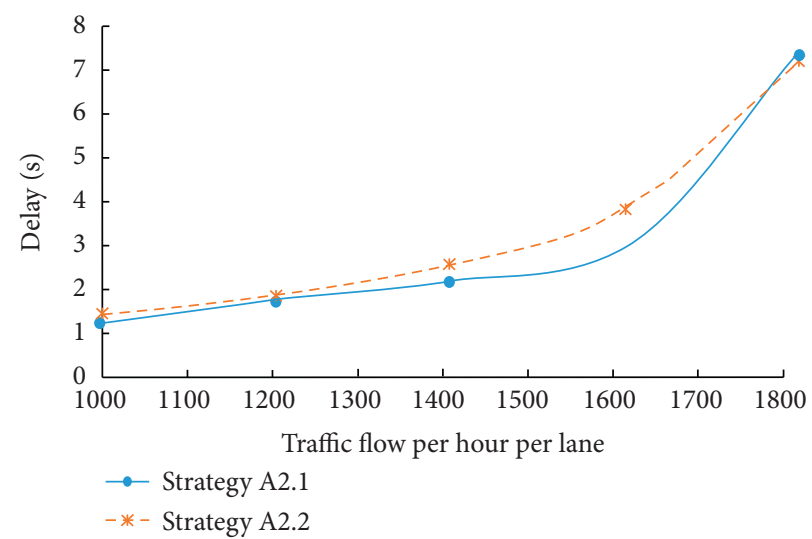

(a)

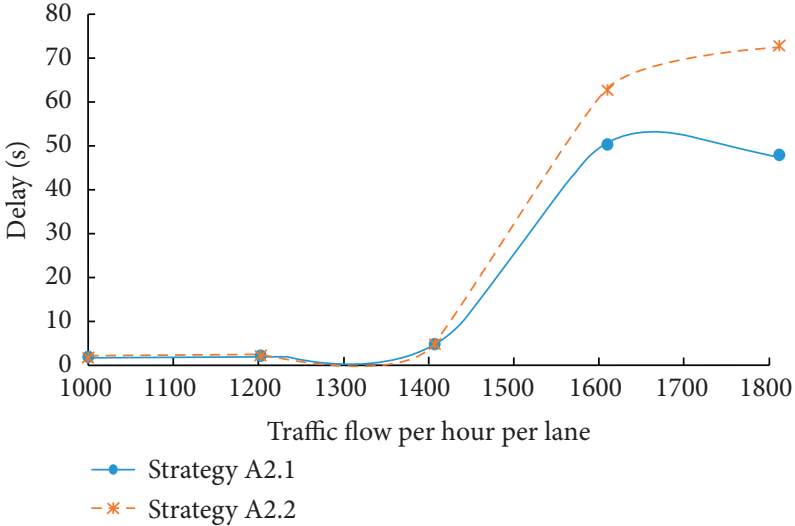

(b)

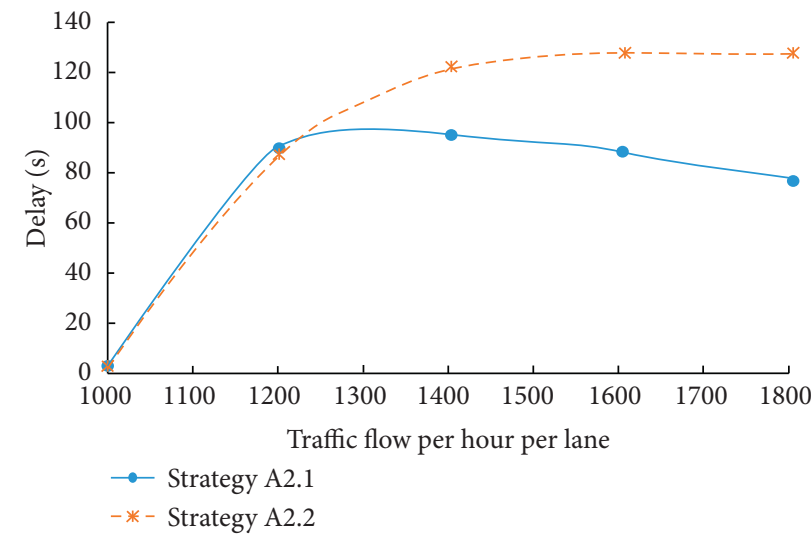

(c)

Figure 8: The average vehicle delay change curves of strategy A2.1 and strategy A2.2 at different off-ramp flow. (a) 20\%. (b) $30 \%$. (c) $40 \%$.

TABLE 1: Actual traffic flow through detection nodes in different simulation conditions (main road total traffic flow and off-ramp traffic flow).

\begin{tabular}{|c|c|c|c|c|c|c|}
\hline \multirow{2}{*}{\multicolumn{2}{|c|}{$\begin{array}{c}\text { Traffic flow per hour per } \\
\text { lane }\end{array}$}} & \multicolumn{5}{|c|}{ Main road } \\
\hline & & 1000 & 1200 & 1400 & 1600 & 1800 \\
\hline \multirow{3}{*}{ Off-ramp rate } & $20 \%$ & $(2953,575)$ & $(3566,691)$ & $(4166,806)$ & $(4763,927)$ & $(5340,1054)$ \\
\hline & $30 \%$ & $(2953,916)$ & $(3566,1082)$ & $(4166,1245)$ & $(4368,1272)$ & $(4281,1256)$ \\
\hline & $40 \%$ & $(2953,1236)$ & $(3320,1317)$ & $(3271,1307)$ & $(3248,1304)$ & $(3341,1325)$ \\
\hline
\end{tabular}

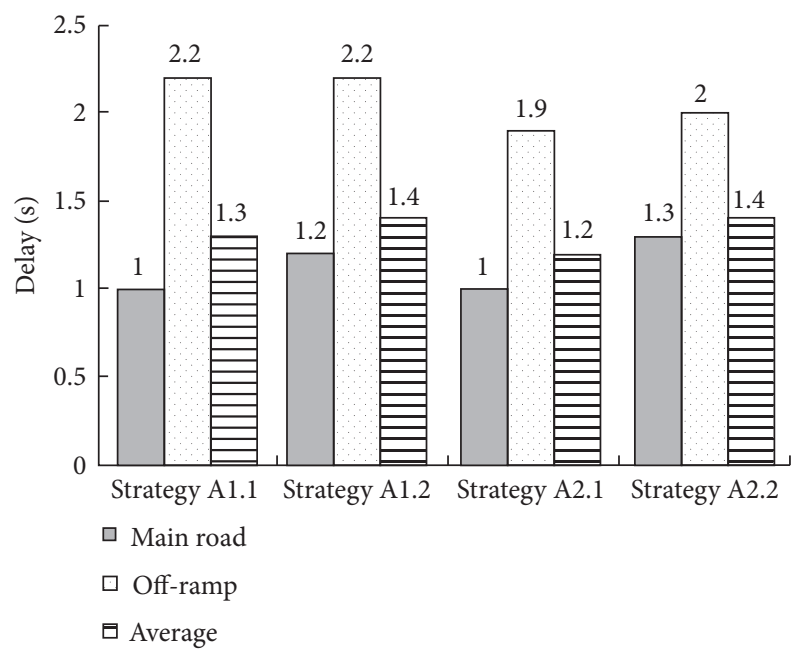

Figure 9: Vehicle delays comparison with main road flow $1000 \mathrm{veh} / \mathrm{h} / \mathrm{lane}$ and off-ramp rate $20 \%$.

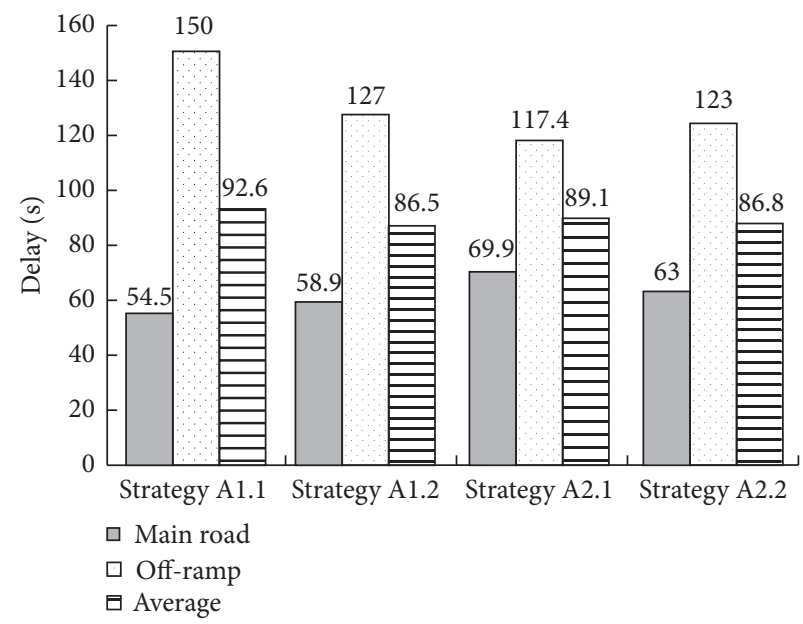

Figure 10: Vehicle delays comparison with main road flow $1200 \mathrm{veh} / \mathrm{h} /$ lane and off-ramp rate $40 \%$. 


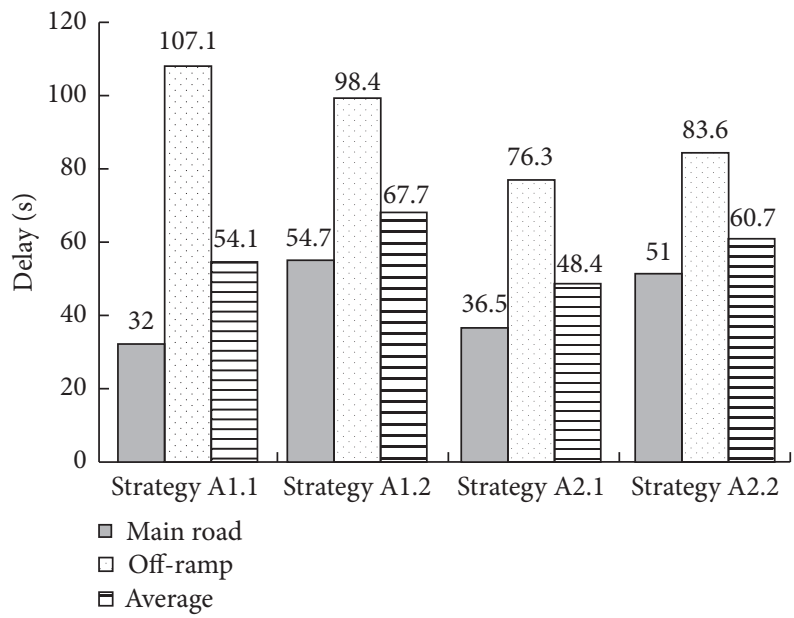

Figure 11: Vehicle delay comparison with main road flow $1600 \mathrm{veh} / \mathrm{h} / \mathrm{lane}$ and off-ramp rate $30 \%$.

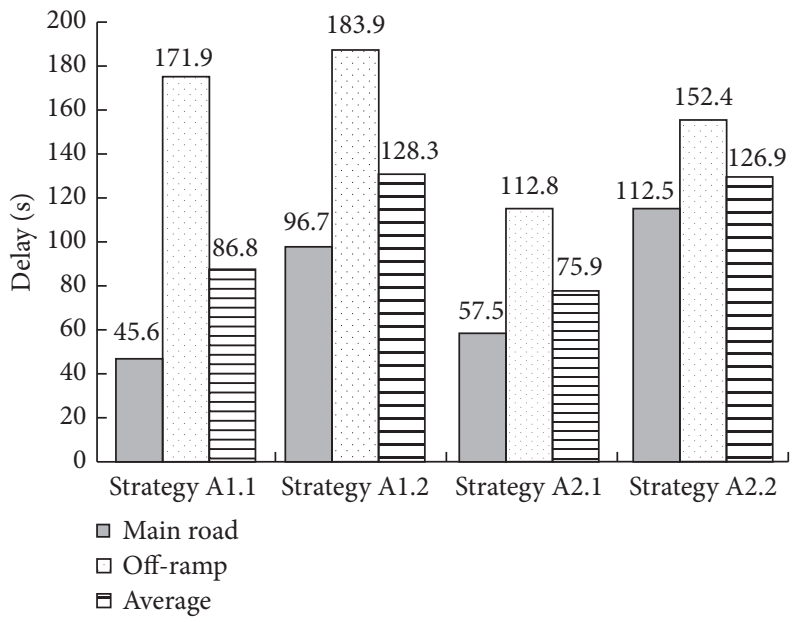

Figure 12: Vehicle delays comparison with main road flow $1800 \mathrm{veh} / \mathrm{h} / \mathrm{lane}$ and off-ramp rate $40 \%$.

green columnar traffic flow state is $\mathrm{A} 1.1=\mathrm{A} 1.2=\mathrm{A} 2.1=\mathrm{A} 2.2$; the optimal strategy in the dark green columnar traffic flow state is $\mathrm{A} 1.2=\mathrm{A} 2.2>\mathrm{A} 2.1>\mathrm{A} 1.1$; the optimal strategy in the yellow columnar traffic flow state is A1.1 > A2.1 > A1.2 > A2.2; the optimal strategy in the brown columnar traffic flow state is A1.1 $>$ A1.2 $>$ A2.2 > A2.1; the optimal strategy in the pink columnar traffic flow state is A2.1 > A1.1 > A1.2>A2.2; and the optimal strategy in the red columnar traffic flow state is $\mathrm{A} 2.1>\mathrm{A} 1.1>\mathrm{A} 2.2>\mathrm{A} 1.2$.

The results show that, in the case of heavy traffic flow, flow-balance strategies for downstream lanes where offramp vehicles merge with the outside lane in advance are more advantageous. When many vehicles are leaving the main road, if the traffic flow is heavy, the flow-balance strategies for lanes where off-ramp vehicles merge with the outside lane in advance (e.g., 0:0:1) are better; otherwise, when the traffic flow on the main road of the off-ramp is relatively small, the flow-balance strategies for lanes where off-ramp vehicles are distributed in lanes with different ratios are better (e.g., $1: 3: 6$ ).

Even though we do not propose a dynamic model for the variation of road traffic flow, the end of this paper presents a table to select the best strategy under different traffic flows, which can preliminarily realize the optimization strategy corresponding to the vehicle flow in most traffic situations. In addition, in the future, we can combine the actual traffic flow situation on the road; for example, the actual 5-minute flow change on a certain ramp can be input into the simulation system built with VISSIM, and then we can get the corresponding strategy in real-time according to traffic flow change.

The focus of this paper is to discuss the macroscopic traffic flow performance in the off-ramp area. But in the future, the foothold will be the microscopic driving behavior of individual vehicles, because the realization of macroscopic optimization requires the specific behavior of vehicles to 
TABLE 2: The comparative analysis table of four strategies under different traffic flow states.

\begin{tabular}{|c|c|c|c|c|c|c|}
\hline \multicolumn{2}{|l|}{$\begin{array}{l}\text { Traffic flow per } \\
\text { hour per lane }\end{array}$} & \multicolumn{5}{|c|}{$\begin{array}{c}\text { Main road } \\
1400\end{array}$} \\
\hline \multirow{3}{*}{ Off-ramp rate } & $20 \%$ & $\begin{array}{c}\mathrm{A} 1.1=\mathrm{A} 1.2= \\
\mathrm{A} 2.1=\mathrm{A} 2.2\end{array}$ & $\begin{array}{c}\mathrm{A} 1.1=\mathrm{A} 1.2= \\
\mathrm{A} 2.1=\mathrm{A} 2.2\end{array}$ & $\begin{array}{c}\mathrm{A} 1.1>\mathrm{A} 2.1> \\
\mathrm{A} 1.2>\mathrm{A} 2.2\end{array}$ & $\begin{array}{c}\mathrm{A} 1.1>\mathrm{A} 2.1> \\
\mathrm{A} 1.2>\mathrm{A} 2.2\end{array}$ & $\begin{array}{l}\mathrm{A} 1.1>\mathrm{A} 1.2> \\
\mathrm{A} 2.2>\mathrm{A} 2.1\end{array}$ \\
\hline & $30 \%$ & $\begin{array}{c}\mathrm{A} 1.1=\mathrm{A} 1.2= \\
\mathrm{A} 2.1=\mathrm{A} 2.2\end{array}$ & $\begin{array}{c}\mathrm{A} 1.1=\mathrm{A} 1.2= \\
\mathrm{A} 2.1=\mathrm{A} 2.2\end{array}$ & $\begin{array}{c}\mathrm{A} 1.1>\mathrm{A} 2.1> \\
\mathrm{A} 1.2>\mathrm{A} 2.2\end{array}$ & $\begin{array}{c}\mathrm{A} 2.1>\mathrm{A} 1.1> \\
\mathrm{A} 2.2>\mathrm{A} 1.2\end{array}$ & $\begin{array}{c}\mathrm{A} 2.1>\mathrm{A} 1.1> \\
\mathrm{A} 2.2>\mathrm{A} 1.2\end{array}$ \\
\hline & $40 \%$ & $\begin{array}{c}\mathrm{A} 1.2=\mathrm{A} 2.2> \\
\mathrm{A} 2.1>\mathrm{A} 1.1\end{array}$ & $\begin{array}{l}\mathrm{A} 1.2=\mathrm{A} 2.2> \\
\mathrm{A} 2.1>\mathrm{A} 1.1\end{array}$ & $\begin{array}{c}\mathrm{A} 2.1>\mathrm{A} 1.1> \\
\mathrm{A} 1.2>\mathrm{A} 2.2\end{array}$ & $\begin{array}{c}\mathrm{A} 2.1>\mathrm{A} 1.1> \\
\mathrm{A} 1.2>\mathrm{A} 2.2\end{array}$ & $\begin{array}{c}\mathrm{A} 2.1>\mathrm{A} 1.1> \\
\mathrm{A} 2.2>\mathrm{A} 1.2\end{array}$ \\
\hline
\end{tabular}

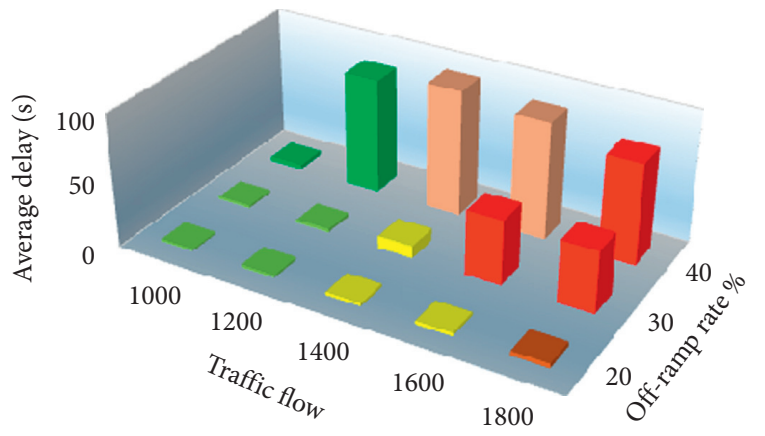

Figure 13: Three-dimensional bar chart of optimal average vehicle delays for different traffic flow conditions.

support. For vehicle control in the connected vehicle environment, we envision that in the future it may be possible either to give instructions to the driver or to control the vehicle directly through a central system.

\section{Conclusions}

(1) Given the expressway off-ramp congestion phenomenon, the traffic flow characteristics and driver lane-changing behaviors in off-ramp areas are analyzed, which provides a theoretical foundation for the vehicle group cooperation strategy under a connected vehicle environment.

(2) Various collaborative strategies of vehicle groups are suggested based on the lateral spatial distributions of traffic flows and geometric lengths of deceleration lanes. Through VISSIM simulation, different strategies are compared and discussed. The merits, shortcomings, and applicable ranges of each strategy are emphasized.

(3) When vehicles are leaving the main road, if traffic flow is heavy, the flow-balance strategy for lanes where off-ramp vehicles merge with the outside lane in advance (for example, the proportion of off-ramp vehicles in the three lanes is 0:0:1) is better; otherwise, when the traffic flow on the main road is relatively small, the flow-balance strategy for lanes where off-ramp vehicles are distributed in lanes with different ratios (e.g., 1:3:6) is better.

(4) What is more, collaborative vehicle lane-changing strategies with different traffic flow states can help to enhance traffic efficiency for connected vehicle environment in the future.

\section{Data Availability}

The data used to support the findings of this study are included within the article.

\section{Conflicts of Interest}

The authors declare that there are no conflicts of interest regarding the publication of this paper.

\section{Acknowledgments}

This work was supported by the Beijing Natural Science Foundation (no. 9202001).

\section{References}

[1] A. Kotsialos and M. Papageorgiou, "Motorway network traffic control systems," European Journal of Operational Research, vol. 152, no. 2, pp. 321-333, 2004.

[2] M. Kontorinaki, I. Karafyllis, and M. Papageorgiou, "Local and coordinated ramp metering within the unifying framework of an adaptive control scheme," Transportation Research Part A: Policy and Practice, vol. 128, pp. 89-113, 2019.

[3] X. Yang, Y. Cheng, and G.-L. Chang, "Integration of adaptive signal control and freeway off-ramp priority control for commuting corridors," Transportation Research Part C: Emerging Technologies, vol. 86, pp. 328-345, 2018.

[4] M. H. Ma, Q. F. Yang, S. D. Liang, and W. Du, "Coordination control of mainline control and ramp metering in freeway merging area(in Chinese)," Journal of Harbin Engineering University, vol. 36, no. 12, pp. 1603-1608, 2015.

[5] C. Pasquale, S. Sacone, S. Siri, and B. De Schutter, "A multiclass model-based control scheme for reducing congestion and emissions in freeway networks by combining ramp metering and route guidance," Transportation Research Part C: Emerging Technologies, vol. 80, pp. 384-408, 2017.

[6] J. Zhao, W. Ma, Y. Liu, and K. Han, "Optimal operation of freeway weaving segment with combination of lane assignment and on-ramp signal control," Transportmetrica A: Transport Science, vol. 12, no. 5, pp. 413-435, 2016.

[7] Z. D. Zhu, S. H. Chen, Y. Q. Yang, A. X. Hu, and X. Y. Zheng, "VISSIM simulation based expressway exit control modes research," Procedia Engineering, vol. 137, pp. 738-746, 2016.

[8] J. Zhao, W. J. Ma, and H. J. Xu, "Increasing the capacity of the intersection downstream of the freeway off-ramp using 
presignals," Computer-Aided Civil and Infrastructure Engineering, vol. 32, no. 8, pp. 674-690, 2017.

[9] D. Sulejic, R. Jiang, N. R. Sabar, and E. Chung, "Optimization of lane-changing distribution for a motorway weaving segment," Transportation Research Procedia, vol. 21, pp. 227-239, 2017.

[10] X. An, J. Zhao, X. D. Ma, and X. M. Xia, "Analysis of lane allocation problem in weaving area based on cellular automaton model(in Chinese)," Application Research of Computers, vol. 36, no. 6, pp. 1656-1659, 2019.

[11] A. Spiliopoulou, M. Kontorinaki, I. Papamichail, and M. Papageorgiou, "Real-time route diversion control at congested freeway off-ramp areas," Transportation Research Part A: Policy and Practice, vol. 107, pp. 90-105, 2018.

[12] J.-W. Zeng, Y.-S. Qian, S.-B. Yu, and X.-T. Wei, "Research on critical characteristics of highway traffic flow based on three phase traffic theory," Physica A: Statistical Mechanics and Its Applications, vol. 530, p. 121567, 2019.

[13] N. Lyu, Y. Cao, C. Wu, J. Xu, and L. Xie, "The effect of gender, occupation and experience on behavior while driving on a freeway deceleration lane based on field operational test data," Accident Analysis \& Prevention, vol. 121, pp. 82-93, 2018.

[14] J. Gong, X. Guo, S. Dai, and L. Duan, "Research on the operational characteristics of diverging area on expressway off-ramp based on different restricted strategy of lane," Procedia-Social and Behavioral Sciences, vol. 96, pp. 11561164, 2013.

[15] J. Shi and M. Liu, "Impacts of differentiated per-lane speed limit on lane changing behaviour: a driving simulator-based study," Transportation Research Part F: Traffic Psychology and Behaviour, vol. 60, pp. 93-104, 2019.

[16] M. Yun, J. Zhao, X. Weng, and X. Yang, "Impact of in-vehicle navigation information on lane-change behavior in urban expressway diverge segments," Accident Analysis and Prevention, vol. 106, pp. 53-66, 2017.

[17] P. Hidas, "Modelling lane changing and merging in microscopic traffic simulation," Transportation Research Part C: Emerging Technologies, vol. 10, no. 5-6, pp. 351-371, 2002.

[18] H. Li, Z. Huang, L. Qin, S. Zheng, and Y. Yang, "Modeling and simulation of vehicle group lane-changing behaviors in upstream segment of ramp areas under a connected vehicle environment," Smart and Resilient Transport, vol. 1, no. 1, pp. 17-29, 2019.

[19] Z. F. Huang, Z. H. Zhang, H. J. Li, L. Q. Qin, and J. Rong, "Determining appropriate lane-changing spacing for offramp areas of urban expressways," Sustainability, vol. 11, no. 7, 2019.

[20] R. Scarinci, A. Hegyi, and B. Heydecker, "Definition of a merging assistant strategy using intelligent vehicles," Transportation Research Part C: Emerging Technologies, vol. 82, pp. 161-179, 2017.

[21] Y. Wang, P. Cai, and G. Lu, "Cooperative autonomous traffic organization method for connected automated vehicles in multi-intersection road networks," Transportation Research Part C: Emerging Technologies, vol. 111, pp. 458-476, 2020.

[22] X. Chang, H. J. Li, J. Rong, X. H. Zhao, and A. R. Li, “Analysis on traffic stability and capacity for mixed traffic flow with platoons of intelligent connected vehicles," Physica A: Statistical Mechanics and Its Applications, vol. 557, no. 2018, Article ID 124829, 2020.

[23] A. Soni and H. Hu, "Formation control for a fleet of autonomous ground vehicles: a survey," Robotics, vol. 7, no. 67, pp. 1-25, 2018.
[24] Y. Li, C. Tang, S. Peeta, and Y. Wang, "Nonlinear consensusbased connected vehicle platoon control incorporating carfollowing interactions and heterogeneous time delays," IEEE Transactions on Intelligent Transportation Systems, vol. 20, no. 6, pp. 2209-2219, 2019.

[25] C. Zhang, N. R. Sabar, E. Chung, A. Bhaskar, and X. Guo, "Optimisation of lane-changing advisory at the motorway lane drop bottleneck," Transportation Research Part C: Emerging Technologies, vol. 106, pp. 303-316, 2019.

[26] J. Tanimoto and X. An, "Improvement of traffic flux with introduction of a new lane-change protocol supported by Intelligent Traffic System," Chaos, Solitons \& Fractals, vol. 122, pp. 1-5, 2019.

[27] Y. Qian, J. Zeng, N. Wang, J. Zhang, and B. Wang, "A traffic flow model considering influence of car-following and its echo characteristics," Nonlinear Dynamics, vol. 89, no. 2, pp. 1099-1109, 2017.

[28] C. Letter and L. Elefteriadou, "Efficient control of fully automated connected vehicles at freeway merge segments," Transportation Research Part C: Emerging Technologies, vol. 80, pp. 190-205, 2017.

[29] Z. H. Khattak, B. L. Smith, H. Park, and M. D. Fontaine, "Cooperative lane control application for fully connected and automated vehicles at multilane freeways," Transportation Research Part C: Emerging Technologies, vol. 111, pp. 294-317, 2020.

[30] S. Zheng, H. Li, Z. Huang, K. Li, and L. Qin, "Flow-balancedbased collaborative strategies and simulation of vehicle group behaviors for on-ramp areas," Simulation Modelling Practice and Theory, vol. 103, Article ID 102093, 2020. 